\title{
Why do cosmological perturbations look classical to us?
}

\author{
Claus Kiefer* \\ Institut für Theoretische Physik, Universität zu Köln, Zülpicher Strasse 77, 50937 Köln, Germany \\ David Polarsk团 \\ Laboratoire de Physique Théorique et Astroparticules, CNRS, \\ Université de Montpellier II, 34095 Montpellier, France
}

\begin{abstract}
According to the inflationary scenario of cosmology, all structure in the Universe can be traced back to primordial fluctuations during an accelerated (inflationary) phase of the very early Universe. A conceptual problem arises due to the fact that the primordial fluctuations are quantum, while the standard scenario of structure formation deals with classical fluctuations. In this essay we present a concise summary of the physics describing the quantum-to-classical transition. We first discuss the observational indistinguishability between classical and quantum correlation functions in the closed system approach (pragmatic view). We then present the open system approach with environmentinduced decoherence. We finally discuss the question of the fluctuations' entropy for which, in principle, the concrete mechanism leading to decoherence possesses observational relevance.
\end{abstract}

Keywords: primordial fluctuations; inflation; decoherence; entropy

\section{INTRODUCTION}

It is often emphasized these days that the field of cosmology has entered a golden age. There is no doubt that the main reason for this statement is the accumulation of observations of ever increasing accuracy. In this way cosmological models aiming to describe the evolution of the Universe from the Big Bang until today are no longer purely speculative: their predictions can be tested and some models can indeed be ruled out.

With the advent of inflationary models, according to which the Universe underwent a phase of accelerated expansion at a very early stage, we now have at our disposal theoretical tools to apprehend such fundamental problems as the origin of cosmological perturbations and the eventual formation of large-scale structures like galaxies. There are many ways in which inflationary models address fundamental physical theories. As inflation is supposed to take place at very high energies in the early Universe, these models offer a unique window on energy scales of the order of $10^{15} \mathrm{GeV}$. Another intriguing aspect of these models is that inflationary perturbations originate from quantum fluctuations though we do not see this quantum nature in the Universe nowadays. It is this aspect of inflationary perturbations that we want to describe in our essay.

We could, of course, as well consider non-inflationary cosmological models in which perturbations are assumed to be classical from the beginning on. However, such models are plagued with problems of causality as distant points on the last-scattering surface, about 350.000 years after the Big Bang, were never in contact before. Hence the impressive homogeneity of the Cosmic Mi-

\footnotetext{
${ }^{*}$ Electronic address: kiefer@thp.uni-koeln.de

${ }^{\dagger}$ Electronic address: polarski@lpta.univ-montp2.fr
}

crowave Background (CMB) would have to be put in by hand in the absence of an inflationary stage. Inflationary models are thus much more natural - and they can be observationally tested.

The main part of our essay consists of four parts. We shall first give in Sec. II a brief review of inflationary cosmology and its mechanism for the generation of perturbations. We then discuss in Sec. III the quantumto-classical transition in the closed system approach (we call it also the pragmatic view) which focusses on the indistinguishability of quantum expectation values and classical stochastic averages. Sec. IV presents the successful observational predictions which emerge from this scenario. Sec. V, then, is devoted to environmental decoherence. We discuss the problem of the classical variables (the pointer basis) as well as the entropy of the fluctuations and its observational significance. We end with a brief conclusion.

\section{INFLATION}

We give here a brief review of the way in which inflationary models give an elegant solution to many fundamental problems occuring in non-inflationary Big-Bang cosmology, see, for example, $\stackrel{1}{.}$. As we shall see, these models do also make characteristic predictions, by which we mean that in the absence of certain observable signatures most if not all inflationary models would be ruled out. We shall first describe the evolution of the homogeneous background for inflation and then turn to the generation of perturbations. 


\section{A. Background expansion}

The crucial point here is that inflation is a stage of accelerated expansion. In this stage, proper (physical) scales are stretched by a huge factor so that scales inside the Hubble radius during inflation will eventually end up at the end of inflation far outside the Hubble radius. Today these scales can correspond to cosmological scales, and typically scales corresponding to the Hubble radius today have exited the Hubble radius during inflation about 65 e-folds before the end of inflation. Typically, inflationary stages are quasi-de Sitter stages during which the Hubble parameter is nearly constant. As we shall see below, inflation provides a mechanism for the causal generation of perturbations.

It is a basic assumption that our Universe is on large scales homogeneous and isotropic. The metric is of the form

$$
d s^{2}=d t^{2}-a^{2}(t)\left[\frac{d r^{2}}{1-k r^{2}}+r^{2}\left(d \theta^{2}+\sin ^{2} \theta d \phi^{2}\right)\right]
$$

a spatially flat universe corresponds to $k=0$, a closed universe to $k=1$, and an open universe to $k=-1$. (We set the speed of light $c=1$ throughout.) In an expanding universe, the scale factor $a(t)$ is a growing function of time, which starts close to zero at the Big Bang about 13.7 billions years ago. The dynamics of the scale factor is given by the Friedmann equations,

$$
\begin{aligned}
\left(\frac{\dot{a}}{a}\right)^{2} & =\sum_{i} \frac{8 \pi G}{3} \rho_{i}-\frac{k}{a^{2}}, \\
\frac{\ddot{a}}{a} & =-\frac{4 \pi G}{3} \sum_{i}\left(\rho_{i}+3 p_{i}\right),
\end{aligned}
$$

where the index $i$ stands for any isotropic (comoving) perfect fluid. For radiation we have $p_{\mathrm{r}}=\rho_{\mathrm{r}} / 3$, for dust $p_{\mathrm{m}}=0$. For the recent accelerated expansion caused by some smooth dark energy component we would have $p_{\mathrm{DE}}=w_{\mathrm{DE}} \rho_{\mathrm{DE}}$, where $w_{\mathrm{DE}}<-1 / 3$ is still unknown and in many models time-dependent. ¿From (3) the expansion is typically decelerated, $\ddot{a}<0$, unless at least one of the components satifies $\rho_{i}+3 p_{i}<0$.

A space-independent scalar field $\phi(t)$ can be viewed as a comoving perfect fluid with

$$
\begin{aligned}
\rho_{\phi} & =\frac{1}{2} \dot{\phi}^{2}+V(\phi), \\
p_{\phi} & =\frac{1}{2} \dot{\phi}^{2}-V(\phi) .
\end{aligned}
$$

Hence, a scalar field $\phi(t)$ can induce an accelerated expansion provided

$$
\dot{\phi}^{2}<V(\phi)
$$

The field $\phi(t)$ driving the inflationary stage is called the inflaton and evolves according to the Klein-Gordon equation

$$
\ddot{\phi}+3 H \dot{\phi}+\frac{d V}{d \phi}=0
$$

which is the form taken by the conservation of energy for a perfect fluid defined by (4) and (5), and we have introduced the Hubble parameter $H \equiv \dot{a} / a$. In most inflationary models, the inflaton field $\phi(t)$ satisfies the slow-roll conditions $\ddot{\phi} \ll 3 H \dot{\phi}$, and hence

$$
3 H \dot{\phi} \approx-\frac{d V}{d \phi}
$$

It is easy to show that the conditions for slow-roll to hold are

$$
\dot{H} \ll 3 H^{2}, \quad \frac{d^{2} V}{d \phi^{2}} \ll 9 H^{2}
$$

in which case the condition (6) is amply satisfied so that accelerated expansion - inflation - takes place.

We conclude this brief summary on the background evolution during inflation by discussing the relative evolution of physical scales. The Hubble radius $R_{\mathrm{H}} \equiv H^{-1}$ defines an important scale in cosmology. If $a \propto t^{p}$, we have $R_{\mathrm{H}} \propto t$, and it is clear that $R_{\mathrm{H}}$ grows faster than a physical scale $\lambda \propto a$ during a decelerated expansion, which has $p<1$. Hence physical scales greater than the Hubble radius, which we shall call "superhorizon" or "super-Hubble" scales, will eventually enter the Hubble radius, by which we mean that they will become smaller than $R_{\mathrm{H}}$ : this is the situation in standard cosmology. This picture changes dramatically during inflation; to illustrate this we take a purely de Sitter stage, which is characterized by $H=$ constant and $a(t) \propto \exp (H t)$. Now it is clear that physical scales inside the Hubble radius, which we shall call "subhorizon" or "sub-Hubble" scales will eventually become larger than the Hubble radius.

If a scale is said to cross the "horizon" 65 e-folds before the end of inflation, this means that at the end of inflation (where $t=t_{\mathrm{e}}$ ) one has $a=a_{\mathrm{e}}=\mathrm{e}^{65} a_{k}$ or $N_{k}=65$ with

$$
N_{k}=\frac{a_{\mathrm{e}}}{a_{k}}
$$

here, $a_{k} \equiv a\left(t_{k}\right)$ if $t_{k}$ is the "horizon-crossing" time of that particular scale with physical wavelength $(2 \pi / k) a$. (Sometimes the factor $2 \pi$ is omitted.) In a pure de Sitter stage this would mean that $H\left(t_{\mathrm{e}}-t_{k}\right)=65$. If we can compute the present physical scale evolving from the Hubble radius during inflation, we know to which physical scale today a scale with given $N_{k}$ corresponds. Depending on the details of the model, the Hubble radius today corresponds typically to a scale with $N_{k} \approx 65$. It can be shown that in slow-roll models $N_{k}$ can be computed from the value $\phi\left(t_{k}\right)$ and that it depends on the potential $V(\phi)$.

In consistent inflationary scenarios, inflation is followed by a standard cosmic expansion during which scales that went outside $R_{\mathrm{H}}$ become again smaller than $R_{\mathrm{H}}$; they "re-enter the horizon". For a given scale, the number of e-folds between the first horizon crossing time $t_{k}$ during inflation and the second horizon crossing time 
during the radiation or matter stage at $t=t_{k, \mathrm{f}}$ is given by the parameter $r_{k}$,

$$
r_{k} \equiv \ln \frac{a\left(t_{k, \mathrm{f}}\right)}{a_{k}} \equiv \ln \frac{a_{k, \mathrm{f}}}{a_{k}} .
$$

We shall see in Sec. III that $r_{k}$ coincides with the squeezing parameter for a quantum state ${ }^{2}$. For typical cosmological scales today, $r_{k} \sim 100$ and even larger. Physically this corresponds to an enormous expansion of the universe, while a given scale $k$ was outside the Hubble radius. As we shall see below, the ensuing huge amount of squeezing for the quantum state plays a crucial role in the quantum-to-classical transition of inflationary quantum fluctuations. It also means that the quantum state originating from inflation is a very peculiar one.

\section{B. Generation of perturbations}

During an inflationary stage, quantum field fluctuations evolve according to the general principles of quantum field theory. Inflation is supposed to take place at an energy scale where space-time can be described as a classical curved space-time on which the quantum field fluctuations are defined. The inflaton fluctuations $\delta \phi(\mathbf{x}, t)$ can be treated as a massless scalar field. This is an excellent approximation when the inflaton field satisfies the slow-roll conditions (9) and it is even exact when we consider primordial gravitational waves.

It is convenient to consider the rescaled quantity $a \delta \phi \equiv$ $y(\mathbf{x}, t)$ and to work with conformal time $\eta=\int d t / a(t)$; a prime will be used to denote a derivative with respect to $\eta$. The formalism presented here is exact for gravitational waves, but can be extended in a straightforward way to the primordial density perturbations.

The quantization of the real perturbation $y(\mathbf{x}, \eta)$ proceeds with the usual canonical quantization scheme. We start from the classical Hamiltonian describing the perturbations,

$$
\begin{aligned}
H & \equiv \int d^{3} \mathbf{x} \mathcal{H}\left(y, p, \partial_{i} y, \eta\right) \\
& =\frac{1}{2} \int d^{3} \mathbf{k}\left[p(\mathbf{k}) p^{*}(\mathbf{k})+k^{2} y(\mathbf{k}) y^{*}(\mathbf{k})\right. \\
& \left.+\frac{a^{\prime}}{a}\left(y(\mathbf{k}) p^{*}(\mathbf{k})+p(\mathbf{k}) y^{*}(\mathbf{k})\right)\right],
\end{aligned}
$$

where $p$ is the momentum conjugate to $y$,

$$
p \equiv \frac{\partial \mathcal{L}\left(y, y^{\prime}\right)}{\partial y^{\prime}}=y^{\prime}-\frac{a^{\prime}}{a} y
$$

In (13) we have introduced the (time-dependent) Fourier transform $y(\mathbf{k}, \eta)$ of the rescaled fluctuation $y(\mathbf{x}, \eta)$. (We sometimes keep the dependence on $\eta$.) In the Lagrangian formulation, it obeys the following classical equation of motion:

$$
y^{\prime \prime}(\mathbf{k}, \eta)+\left(k^{2}-\frac{a^{\prime \prime}}{a}\right) y(\mathbf{k}, \eta)=0 .
$$

Upon quantization, the Fourier transforms are promoted to operators on which we impose the canonical commutation relations,

$$
\left[y(\mathbf{k}, \eta), p^{\dagger}\left(\mathbf{k}^{\prime}, \eta\right)\right]=\mathrm{i} \delta^{(3)}\left(\mathbf{k}-\mathbf{k}^{\prime}\right) .
$$

(We set $\hbar=1$.) We can write the Hamiltonian operator in the following way:

$$
\begin{aligned}
H= & \int \frac{d^{3} \mathbf{k}}{2}\left[k\left(a(\mathbf{k}) a^{\dagger}(\mathbf{k})+a^{\dagger}(-\mathbf{k}) a(-\mathbf{k})\right)+\right. \\
& \left.\mathrm{i} \frac{a^{\prime}}{a}\left(a^{\dagger}(\mathbf{k}) a^{\dagger}(-\mathbf{k})-a(\mathbf{k}) a(-\mathbf{k})\right)\right] .
\end{aligned}
$$

The time-dependent annihilation operators $a(\mathbf{k})$ (we often skip the argument $\eta$ for conciseness) appearing in (17) are defined as usual,

$$
a(\mathbf{k})=\frac{1}{\sqrt{2}}\left(\sqrt{k} y(\mathbf{k})+\frac{\mathrm{i}}{\sqrt{k}} p(\mathbf{k})\right),
$$

so that

$$
\begin{aligned}
& y(\mathbf{k})=\frac{a(\mathbf{k})+a^{\dagger}(-\mathbf{k})}{\sqrt{2 k}}, \\
& p(\mathbf{k})=-\mathrm{i} \sqrt{\frac{k}{2}}\left(a(\mathbf{k})-a^{\dagger}(-\mathbf{k})\right) .
\end{aligned}
$$

It is easily seen from (16) that $a$ and $a^{\dagger}$ satisfy the commutation relations

$$
\left[a(\mathbf{k}, \eta), a^{\dagger}\left(\mathbf{k}^{\prime}, \eta\right)\right]=\delta^{(3)}\left(\mathbf{k}-\mathbf{k}^{\prime}\right) .
$$

Let us consider the time evolution of these operators. ¿From the Hamiltonian (17) we get

$$
\left(\begin{array}{c}
a^{\prime}(\mathbf{k}) \\
\left(a^{\dagger}(-\mathbf{k})\right)^{\prime}
\end{array}\right)=k\left(\begin{array}{cc}
-\mathrm{i} & \frac{a H}{k} \\
\frac{a H}{k} & \mathrm{i}
\end{array}\right)\left(\begin{array}{c}
a(\mathbf{k}) \\
a^{\dagger}(-\mathbf{k})
\end{array}\right) .
$$

The second piece of the Hamiltonian (17), which is proportional to $a^{\prime} / a$, is responsible for a mixing between creation and annihilation operators. In the Heisenberg representation it corresponds to a Bogolubov transformation; physically it means that particles are produced in pairs with opposite momenta. For reasons that will become clear later, this phenomenon is called squeezing in the Schrödinger picture; the corresponding squeezing parameter $r_{k}$ turns out to be given by the expression (11) above. ¿From (22) one can see that mixing of creation and annihilation operators is efficient when the off-diagonal terms dominate, in other words, on superHubble scales when $a H / k \gg 1$.

Using (20) and (22), one obtains after a little algebra,

$$
y(\mathbf{k}, \eta) \equiv f_{k}(\eta) a_{\mathbf{k}}+f_{k}^{*}(\eta) a_{-\mathbf{k}}^{\dagger},
$$

where $a_{\mathbf{k}} \equiv a\left(\mathbf{k}, \eta_{0}\right)$, and the field modes $f_{k}$ obey Equation (15) and satisfy $f_{k}\left(\eta_{0}\right)=1 / \sqrt{2 k}$. At the initial time 
$\eta_{0}$, the field modes are deep inside the Hubble radius. Equation (23) can be written in the suggestive way

$$
y(\mathbf{k}, \eta)=\sqrt{2 k} f_{k 1}(\eta) y_{\mathbf{k}}-\sqrt{\frac{2}{k}} f_{k 2}(\eta) p_{\mathbf{k}},
$$

where $y_{\mathbf{k}} \equiv y\left(\mathbf{k}, \eta_{0}\right)$ and $p_{\mathbf{k}} \equiv p\left(\mathbf{k}, \eta_{0}\right), f_{k 1}=\Re f_{k}, f_{k 2}=$ $\Im f_{k}$. We have in an analogous way momentum modes $g_{k}(\eta)$, with $g_{k}\left(\eta_{0}\right)=\sqrt{k / 2}$,

$$
p(\mathbf{k})=\sqrt{\frac{2}{k}} g_{k 1}(\eta) p_{\mathbf{k}}+\sqrt{2 k} g_{k 2}(\eta) y_{\mathbf{k}} .
$$

We shall now address the first step in understanding why and to which extent these quantum field modes appear classically.

\section{QUANTUM-TO-CLASSICAL TRANSITION: THE PRAGMATIC VIEW}

In the last section we have described the evolution of the quantum modes in the Heisenberg representation, in which operators evolve in time and quantum states do not. While the quantum-to-classical transition is in general formulated in the Schrödinger picture, for the inflationary perturbations the Heisenberg picture provides deep insight, too.

To see this, let us assume that there is a limit in which $f_{k 2}$ and $g_{k 1}$ (or $f_{k 1}$ and $g_{k 2}$ ) vanish. Then it is clear from (24) that the non-commutativity of the operators $y_{\mathbf{k}}$ and $p_{\mathbf{k}}$ is no longer relevant. What is the physical meaning of such a limit? Let us consider a classical stochastic system where the dynamics is still described by equations of the form (24), but with now $y\left(\mathbf{k}, \eta_{0}\right)$ and $p\left(\mathbf{k}, \eta_{0}\right)$ representing random initial values (c-numbers). If $f_{k 2}$ and $g_{k 1}$ vanish, we get

$$
p(\mathbf{k}, \eta) \equiv p_{\mathrm{cl}}(y(\mathbf{k}, \eta))=\frac{g_{k 2}}{f_{k 1}} y(\mathbf{k}, \eta) .
$$

This is true for the quantum system (in the operator sense) and for the classical stochastic system (in the c-number sense). Therefore, for a given realization of the perturbation $y(\mathbf{k}, \eta)$, the corresponding momentum $p_{\mathrm{cl}}(\mathbf{k}, \eta)$ is fixed and equal to the classical momentum corresponding to this value $y(\mathbf{k}, \eta)$. Then the quantum system is effectively equivalent to the classical random system, which is an ensemble of classical trajectories with a certain probability associated to each of them ${ }^{3}$.

This is, in fact, what happens for the primordial fluctuations. The field modes obey (15), and this equation has, on super-Hubble scales, solutions that become dominant and solutions that become negligible (so-called "growing" and "decaying" modes). Eventually the decaying mode can be neglected and one in left with the growing mode. It turns out that $f_{k 2}$ and $g_{k 1}$ are decaying modes, and one is left with (26).

From the Heisenberg representation it follows that the operational equivalence with the classical stochastic system does not depend on the initial state; this was indeed shown explicitly for a wide class of initial states (and extended to some gauge-invariant quantities) 4 .

We now look at the problem in the Schrödinger representation where the state evolves in time, while the operators are fixed. The initial quantum state of the perturbations is the vacuum state $\left|0, \eta_{0}\right\rangle$ satisfying

$$
a_{\mathbf{k}}\left|0, \eta_{0}\right\rangle=0 \quad \forall \mathbf{k} .
$$

At later times, due to the creation of particles, the timeevolved state is annihilated by a more complicated operator,

$$
\left\{y_{\mathbf{k}}+\mathrm{i} \gamma_{k}^{-1}(\eta) p_{\mathbf{k}}\right\}|0, \eta\rangle=0
$$

The corresponding (Gaussian) wave function reads

$$
\begin{aligned}
& \Psi\left[y_{\mathbf{k}}, y_{\mathbf{k}}^{*}, \eta\right]=\frac{1}{\sqrt{\pi\left|f_{k}\right|^{2}}} \exp \left(-\frac{\left|y_{\mathbf{k}}\right|^{2}}{2\left|f_{k}\right|^{2}}\{1-\mathrm{i} 2 F(k)\}\right) \\
& \equiv\left(\frac{2 \Omega_{\mathrm{R}}(\eta)}{\pi}\right)^{1 / 4} \exp \left(-\left[\Omega_{\mathrm{R}}(\eta)+\mathrm{i} \Omega_{\mathrm{I}}(\eta)\right]\left|y_{\mathbf{k}}\right|^{2}\right) .(29)
\end{aligned}
$$

In (28 29), we have

$$
\begin{aligned}
\gamma_{k} & =\frac{1}{2\left|f_{k}\right|^{2}}[1-2 \mathrm{i} F(k)], \\
F(k) & =\Im f_{k}^{*} g_{k}=f_{k 1} g_{k 2}-f_{k 2} g_{k 1} .
\end{aligned}
$$

At the initial time $\eta=\eta_{0}, \gamma_{k}\left(\eta_{0}\right)=k$, and hence $F(k)=0$; in other words, we have a minimum uncertainty wave function. This is no longer so later, as $|F(k)|$ becomes very large; the probabilities, however, remain Gaussian. Another way to exhibit the physical meaning of our state is to consider the Wigner function, $W$, which can be considered as a kind of quasi-probability density in phase space. For Gaussian wave functions, $W$ has the property to be positive definite. For the wave function (29) one obtains

$$
W=\stackrel{\left|r_{k}\right| \rightarrow \infty}{\longrightarrow}|\Psi|^{2} \delta^{(2)}\left(p_{\mathbf{k}}-p_{\mathrm{cl}}\left(y_{\mathbf{k}}\right)\right)
$$

The dynamics of the fluctuations leads to the largesqueezing limit $\left|r_{k}\right| \rightarrow \infty$. One gets a highly elongated ellipse whose large axis is oriented along the line $p_{\mathbf{k}}=p_{\mathrm{cl}}\left(y_{\mathbf{k}}\right)$ and whose width becomes negligible. This is a direct vizualisation of the classical stochastic behaviour of our system: the variable $y_{\mathbf{k}}$ can take any value with corresponding probability $|\Psi|^{2}$, while $p_{\mathbf{k}}$ takes the corresponding value $p_{\mathbf{k}}=p_{\mathrm{cl}}\left(y_{\mathbf{k}}\right)$. Instead of being essentially located in phase space around one physical trajectory, as for coherent states, the system behaves as if it followed an infinite number of classical trajectories with a definite probability to be on each of them. Interestingly, an analogous situation happens for a free non-relativistic particle ${ }^{5}$ possessing an initial Gaussian minimal uncertainty wavefunction. As is well known, $F \propto t$ and becomes very large. At very late times, the position does no longer depend on the initial position,

$$
x(t) \simeq \frac{p_{0}}{m} t .
$$


We get an equivalence with an ensemble of classical particles obeying (32), where $p_{0}$ is a random variable with probability $P\left(p_{0}\right)=|\Psi|^{2}\left(p_{0}\right)$. This illustrates the kind of classicality we are dealing with. Moreover, when (32) holds, position operators at different times approximately commute (which, in quantum-optical language, corresponds to a quantum-nondemolition situation).

Using the canonical commutation relations, the quantum coherence between the growing and decaying mode can be expressed as

$$
f_{k 1} g_{k 1}+f_{k 2} g_{k 2}=\frac{1}{2} .
$$

Clearly, when $f_{k 2}, g_{k 1}$ are unobservable, this coherence becomes unobservable as well. This is the case when the decaying mode is so small that we have no access to it in observations. For the ratio of the growing to the decaying mode one has

$$
\frac{f_{k 2}}{f_{k 1}} \propto e^{-2\left|r_{k}\right|}
$$

which is why a large squeezing parameter $r_{k}$ in the Schrödinger picture implies a vanishing decaying mode in the Heisenberg representation. The width of the Wigner function is given by

$$
\left\langle\left(p_{\mathbf{k}}-p_{\mathrm{cl}}\left(y_{\mathbf{k}}\right)\right)^{2}\right\rangle=g_{k 1}^{2},
$$

which becomes unobservable like the decaying mode. A further consequence is that the typical phase-space volume occupied by the system becomes negligible, too.

Let us take the concrete and important example of a perturbation on de Sitter space $a \propto \mathrm{e}^{H t}$, with $H$ being constant. The exact solution of (15) with the correct initial condition (ground state for initial sub-Hubble modes) then reads up to an unimportant constant phase factor

$$
\begin{aligned}
& f_{k}=\frac{-\mathrm{i}}{\sqrt{2 k}} \mathrm{e}^{-\mathrm{i} k \eta}\left(1-\frac{\mathrm{i}}{k \eta}\right), \\
& g_{k}=-\mathrm{i} \sqrt{\frac{k}{2}} \mathrm{e}^{-\mathrm{i} k \eta}, \quad \eta \equiv-\frac{1}{a H}<0 .
\end{aligned}
$$

Modes initially inside the Hubble radius become much larger than the Hubble radius during inflation solely as a result of their dynamics to satisfy $k \eta \ll 1$ : here we have the limit mentioned above! This can be shown also to correspond to the large-squeezing limit. Actually, this is a particular case of the general situation when an equation like (15) has a growing-mode solution and a decayingmode solution. Here the decaying mode becomes vanishingly small; when it is neglected we are in the limit of a random stochastic process. Perturbations are then given by

$$
\delta \phi(\mathbf{k}, \eta)=\frac{H}{\sqrt{2 k^{3}}} e_{\mathbf{k}} .
$$

We have set here $\sqrt{2 k} y_{\mathbf{k}}=e_{\mathbf{k}}$, which assumes the role of a classical Gaussian random variable with unit variance. From (38) we see that the perturbations tend to a constant value (they become "frozen"). One should realize that the true reason for the quantum-to-classical transition in the sense discussed here is that the decaying mode becomes vanishingly small. Primordial gravitational waves follow exactly the behaviour (38) (up to some factor $)^{6}$, but after re-entering the Hubble radius they will start oscillating. They retain their classical appearance because the decaying mode (which oscillates as well by then!) is negligible ${ }^{3}$.

\section{OBSERVATIONAL PREDICTIONS}

The perturbations produced during inflation have remarkable properties which can be confronted with observations. This confrontation makes essential use of the effective classical behaviour discussed in the last section.

Primordial inflaton fluctuations generate a primordial Newtonian potential and the corresponding energydensity fluctuations $\delta \rho$. A central quantity is the power spectrum, $P(k)$, of the quantity $\delta \equiv \delta \rho / \rho$,

$$
\left\langle\delta(\mathbf{k}) \delta^{*}\left(\mathbf{k}^{\prime}\right)\right\rangle=P(k) \delta^{(3)}\left(\mathbf{k}-\mathbf{k}^{\prime}\right) .
$$

When the statistical properties are isotropic, the power spectrum depends only on $k \equiv|\mathbf{k}|$. It can be shown that the power spectrum is the Fourier transform of the correlation function (in space), and it can be defined for any quantity. Deep in the matter-dominated stage, $P(k)$ has the following expression on "super-horizon" scales in slow-roll single-field inflation,

$$
P(k)=\frac{1024}{75} \pi^{3} G^{3}\left(\frac{V^{3}}{V^{\prime 2}}\right)_{t_{k}}(a H)^{-4} k,
$$

where $V^{\prime}$ is the derivative of the inflaton potential with respect to the inflaton $\phi$, and the fraction has to be evaluated at the Hubble-radius crossing time $k=a\left(t_{k}\right) H\left(t_{k}\right)$ during inflation. Because of the quasi-exponential inflationary expansion, it depends very weakly on $k$. Neglecting this dependence, we get

$$
P(k) \propto k,
$$

which is the scale-invariant "Harrison-Zeldovich" spectrum that plays a crucial role in these investigations. This spectrum is called scale-invariant for the following reason: if we compute the r.m.s. relative mass fluctuations $\left\langle(\delta M / M)^{2}\right\rangle$ at the time $t_{k}$ when a scale eventually re-enters the Hubble radius, the same value is obtained for all scales.

Using the expansion (23) and the commutation relations (21), it is straightforward to show that

$$
\left\langle\delta \phi^{2}\right\rangle=\frac{1}{2 \pi^{2}} \int_{0}^{\infty} d k k^{2}\left|\delta \phi_{k}(\eta)\right|^{2},
$$

with $f_{k}(\eta)=a \delta \phi_{k}(\eta)$. This means that the power spectrum of $\delta \phi$ is just given by $\left|\delta \phi_{k}(\eta)\right|^{2}$. However, the average on the left is a quantum average; it is only by virtue 
of the quantum-to-classical transition mentioned above that we can consider $\left|\delta \phi_{k}(\eta)\right|^{2}$ as the power spectrum of a classical random variable, whose time evolution is consistent with probabilities conserved along classical trajectories. In the opposite case this would be impossible due to quantum interferences. We note also the result in the limit (38), which gives

$$
\frac{d\left\langle\delta \phi^{2}\right\rangle}{d \ln k}=\left(\frac{H}{2 \pi}\right)^{2},
$$

where the derivative is with respect to some cut-off value.

Primordial fluctuations leave their imprint on the $\mathrm{CMB}$ and this provides the best constraint on their properties and on the inflationary models in which they were presumably produced. While the CMB is remarkably homogeneous with a black body spectrum, perturbations induce very tiny inhomegeneities of the order $10^{-5}$. In this regime, linear perturbation theory is very accurate so that precise predictions can be made. The measurement of the temperature anisotropies angular power spectrum, the $C_{\ell}$ 's,

$$
C_{\ell}=\left\langle\left|a_{l m}\right|^{2}\right\rangle, \quad \frac{\Delta T}{T}(\vartheta, \varphi)=\sum_{l, m} a_{l m} Y_{l m},
$$

(which are in the isotropic case independent of $m$ ) will culminate with the Planck satellite (ESA). The exquisite data we have thus far, in particular those collected by the WMAP collaboration (NASA), show excellent agreement with a flat universe and adiabatic perturbations ${ }^{7.8}$. Such perturbations respect the equation of state of the background; for the baryon-photon plasma this is when $\frac{\delta T}{T}=\frac{1}{3} \frac{\delta n_{B}}{n_{B}}$, where $n$ is the baryon number density. This is a natural outcome of single-field inflation.

Before decoupling, the baryon-photon plasma is tightly coupled and its density oscillates on scales inside the Hubble radius, yielding oscillations similar to pressure waves. These are often called acoustic oscillations. The location of the first (Doppler) peak gives roughly the angular scale of the Hubble radius at decoupling and is consistent with a flat universe. The pattern of the angular power spectrum is in agreement with primordial adiabatic fluctuations. After decoupling, the baryons retain the primordially induced acoustic "Sakharov" oscillations, the baryonic acoustic oscillations (BAO); these were detected in the galaxy power spectrum and are presently used in order to constrain dark energy models.

To parametrize the departure from scale invariance, one introduces the spectral index $n$ with $P(k) \propto k^{n}$. Latest CMB data constrain $n$ to be very close, but slightly lower than one $e^{7}$. Finally we see no clear evidence for non-Gaussianity in the statistics of the perturbations. All these data are in surprisingly good agreement with the simplest single-field slow-roll inflationary models (see e.g. $\underline{\underline{9}})$.

Let us return in more detail to the acoustic oscillations. They arise because of the standing-wave behaviour of the perturbations inside the Hubble radius. There are always two modes that are solutions to the equations and they will both oscillate. One of the modes matches the growing (dominant) mode, and the other the decaying (subdominant) mode. For modes sufficiently long outside the Hubble radius, the decaying mode disappears and the growing mode will match the corresponding oscillating mode inside the Hubble radius. At decoupling, each mode has a given oscillation phase, and this gives rise to the acoustic oscillations seen in the $C_{\ell}$ 's. If we had a way to generate classical perturbations that would evolve outside the Hubble radius for very long, just the same would be true. If these perturbations had random initial conditions, obeying the same statistics as our initially quantum fluctuations, both systems would be indistinguishable. Hence the presence of acoustic oscillations is in no way connected to the quantum nature of the perturbations but rather to their primordial origin. But the quantum-to-classical transition can only take place in a system where the decaying mode is negligible enough so that acoustic oscillations $d o$ arise. It is interesting that a similar standing-wave behaviour is present in the primordial stochastic gravitational waves background produced during inflation. Unfortunately, to detect it in a direct detection experiment today would require a resolution in

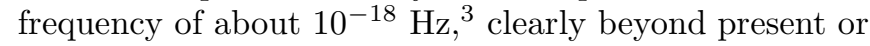
foreseeable capabilities. The same property yields also small superimposed oscillations in the power spectra of the CMB temperature anisotropy and polarization. This is similar to the acoustic oscillations but with a period approximately twice as small (solely due to the difference between the light velocity and the sound velocity in the baryon-photon plasma at the recombination time $)^{3}$. Their observation is very difficult but not hopeless if the parameter characterizing the tensor-to-scalar ratio in the CMB temperature anisotropy is not too small, see $\frac{10}{\underline{10}}$ for detailed estimates of the CMB polarization B-mode produced by primordial gravitational waves only.

We finally mention that calculations done for the creation of matter by parametric resonance after inflation use the description of perturbations in terms of classical stochastic fields. All the predictions mentioned above and which were confirmed by observations are done in the closed-system approach, that is, by taking the perturbations as an isolated system. Similar results were obtained in various disguise by several authors $11,12,13$ and even extended beyond the linear regime $\stackrel{14}{ }$. In this approach the system becomes indistinguishable, in an operational sense, from a classical stochastic system solely by virtue of its peculiar inflationary dynamics.

From a purely pragmatic point of view, the closedsystem approach is sufficient. In astrophysical observations one measures certain classical correlation functions for which the above line of thought shows that they are indistinguishable from the fundamental quantum expectation values. Still, in the next section we shall go beyond the closed-system approach by taking into account the interaction of the modes with other, "environmental", 
degrees of freedom. This has several reasons. First, the environment-induced decoherence process is generally invoked in order to explain the appearance of classical behaviour in quantum theory 15 . Second, since an environment is expected to be present anyway, it is important to consider whether it does not spoil the successful predictions from the closed-system approach. It should, in particular, not erase the acoustic oscillations. Moreover, invoking large non-linear effects might irremediably modify the CMB angular power spectrum and induce large non-Gaussianity. Finally, there is the question about the entropy of the perturbations which by definition cannot be addressed inside the closed-system approach.

We shall see that these questions and problems can be successfully dealt with without spoiling the successful predictions of the closed-system approach including the quantum-to-classical transition in the pragmatic approach adopted in this section.

\section{QUANTUM-TO-CLASSICAL TRANSITION: DECOHERENCE}

\section{A. Decoherence and pointer basis}

In the last section we have described the primordial fluctuations in cosmology by a collection of independent quantum states labelled by the wave number $k$. Since no interaction between different $k$ or between the fluctuations and other fields have been considered, we deal with a pure quantum state for each $k$. The initial condition for each quantum state is the harmonic-oscillator ground state with respect to $k$. During inflation, modes with wavelengths larger than the Hubble scale $H^{-1}$ assume a squeezed Gaussian state. We focussed attention on the modes far outside the Hubble scale, which experience an enormous squeezing. For these highlysqueezed modes, which are the ones relevant for cosmological observations, all expectation values containing the field-amplitudes or their momenta are indistinguishable from classical stochastic averages ${ }^{3}$. It is this approximate coincidence between quantum and classical expectation which is the basis of the pragmatic approach to the quantum-to-classical transition discussed above for the primordial fluctuations.

One can, however, adopt a more fundamental point of view. It is far from realistic to assume that a primordial fluctuation with wave number $k$ is exactly isolated. We must take into account its interaction with other degrees of freedom (called the 'environment' for simplicity). The main reason is the following. As one knows from standard quantum theory, even a tiny interaction with other degrees of freedom can become important, in the sense that an entanglement of a system with its environment can form even without direct disturbance of the system. If the environmental degrees of freedom are inaccessible to observations (as they usually are), the ensuing entanglement with the system leads to decoherence - interference terms can no longer be observed at the system itself and the system appears classical ${ }^{15}$. This is the fundamental origin of the quantum-to-classical transition. The phenomenon of decoherence is by now theoretically well understood and has been experimentally tested with high precision 15,16,17. Decoherence leads to an apparent ensemble of wave packets for the observable with respect to which the interferences vanish. A paradigmatic example is the localization of a quantum particle due to scattering with photons, air molecules, or other particles $15,17,22$. There the position basis of the particle is the approximate basis distinguished by the scattering process. The basis distinguished by the environment is generally called the pointer basis; the corresponding observable is called pointer observable. Interferences between different members of the pointer basis are suppressed by the decohering influence of the environment.

One would expect, therefore, that decoherence is of crucial importance for the primordial fluctuations, too. This expectation is, moreover, supported by the fact that the system by itself evolves into a highly squeezed state in which squeezing is in the field momentum and broadening is in the field amplitude (corresponding to the position variable in quantum mechanics): one knows from quantum theory that highly squeezed states are extremely sensitive to any environment $\frac{15}{2}$. This is the reason why they are so difficult to generate in the laboratory - it is very hard to isolate them from any environment. In view of their huge squeezing, this argument should apply to the cosmological fluctuations a fortiori.

But could it be imaginable that the cosmological fluctuations, in contrast to a typical quantum-mechanical situation, are indeed strictly isolated? The answer is definitely no.

Firstly, in any fundamental theory (such as string theory) there is an abundance of different fields with different interactions. Among them it will not be difficult to find appropriate candidates for environmental fields generating decoherence for the primordial fluctuations.

Secondly, even if one assumes to have no such fields, there are two processes which cannot be neglected. The first one is the interaction between modes with different $k$; recall that the full theory is non-linear and that, therefore, the various modes cannot be treated independently of each other. Such non-linear interactions concern both the interaction with the modes of the inflaton and the perturbations of the metric (containing, in particular, gravitational waves).

The second process is the entanglement of the modes' quantum state between different spatial regions: even if the modes are independent in $k$-space, the Gaussian wave functions for the amplitudes in real space are highly correlated over spacelike regions (as in the EinsteinPodolsky-Rosen situation). This leads, in particular, to an entanglement between the regions inside and outside the Hubble radius. Famous non-cosmological examples are the Hawking and the Unruh effects, where the thermal appearance of the corresponding radiation 
can be understood from the entanglement between inside and outside the event horizons and the tracing out of the correlations into the horizon $\frac{18}{}$. Even for spacelike surfaces which stay outside the horizon, the thermal nature of Hawking and Unruh radiation can be understood from the entanglement with other fields, leading to decoherence ${ }^{19}$.

The process of decoherence is, moreover, needed to justify the results from the isolated (closed) system in the first place. Even if the classical and quantum expectation values are indistinguishable, the presence of a pure state means that one has a quantum superposition of all possible field amplitudes, not an ensemble of stochastically distributed classical values. This situation is similar to Schrödinger's cat. In the pragmatic point of view of Sec. III, the approximate coincidence of the expectation values suffices. Such a coincidence is, however, not sufficient for a realistic interpretation. Only decoherence can eventually justify the pragmatic point of view in that it leads to an apparent ensemble of wave packets for the system variables itself (which, in our case, are the field amplitudes). The insufficience of approximately equal classical and quantum expectation values for a fundamental interpretation has recently been clearly emphasized in a different context (the quantum mechanics of classically chaotic systems) by Schlosshauer ${ }^{20}$. In the presence of a pure state one can always find an observable for which no classical counterpart exists, that is, for which the comparison of quantum and classical expectation values is meaningless.

The quantum-to-classical transition happens for the highly-squeezed modes whose wavelengths exceed the Hubble scale. It is for these modes where environmental decoherence is most efficient $\underline{21}$. How can this happen? Would one not expect that no causal interaction can occur on scales larger than the Hubble scale? This is true only for a direct disturbance of the system. But the crucial point is that quantum entanglement can form without direct disturbance. And this is all one needs for decoherence! In the context of the quantum measurement process, the sole formation of entanglement is referred to as an 'ideal measurement' or a 'quantum non-demolition measurement': the system remains undisturbed, but the environment is affected through the formation of entanglement. The general mechanism is as follows 15 .

Consider a quantum system which is initially in the state $|n\rangle$ and a 'measurement device' (here: the environment) which is in some initial state $\left|\Phi_{0}\right\rangle$. (We assume that $|n\rangle$ belongs to a set of eigenstates of a system observable.) The evolution according to the Schrödinger equation is in the special case of an 'ideal measurement' given by

$$
|n\rangle\left|\Phi_{0}\right\rangle \stackrel{t}{\longrightarrow} \exp \left(-\mathrm{i} H_{\mathrm{int}} t\right)|n\rangle\left|\Phi_{0}\right\rangle=|n\rangle\left|\Phi_{n}(t)\right\rangle,
$$

where $H_{\text {int }}$ denotes the interaction Hamiltonian (assumed here to dominate over the free Hamiltonians) which correlates the system state with its environment without changing the system state.
In the general case, the quantum system can be in a superposition of different eigenstates of the system observable. Then, due to the linearity of the time evolution, an initial product state with $\left|\Phi_{0}\right\rangle$ develops into an entangled state of system plus apparatus,

$$
\left(\sum_{n} c_{n}|n\rangle\right)\left|\Phi_{0}\right\rangle \stackrel{t}{\longrightarrow} \sum_{n} c_{n}|n\rangle\left|\Phi_{n}(t)\right\rangle .
$$

But this is a highly non-classical state! Since the environmental states $\left\{\left|\Phi_{n}\right\rangle\right\}$ are not accessible, they have to be traced out from the full quantum state. One thereby arrives at the reduced density matrix $\rho_{\mathrm{S}}$ which contains all the information that is available at the system itself. Since the environmental states $\left\{\left|\Phi_{n}\right\rangle\right\}$ can be assumed as being approximately orthogonal (otherwise they would not be able to serve as a 'measurement device'), the reduced density matrix is of the form

$$
\rho_{\mathrm{S}} \approx \sum_{n}\left|c_{n}\right|^{2}|n\rangle\langle n|,
$$

that is, it assumes the form of an approximate ensemble for the various system states $|n\rangle$, each of which occurs with probability $\left|c_{n}\right|^{2}$.

In our case, the cosmological fluctuations represent the system to be decohered. The environmental states $\left\{\left|\Phi_{n}\right\rangle\right\}$ can be other fields or inaccessible parts of the fluctuations themselves (see below). The system states $|n\rangle$ are given by the field-amplitude states $\left|y_{\mathbf{k}}\right\rangle$. The interaction with the environment can, in the ideal-measurement case, be described by the multiplication of an initial density matrix $\rho_{0}\left(y, y^{\prime}\right)$ with a Gaussian factor in $y-y^{\prime}$ (omitting here and in the following the index $\mathbf{k}$ in $y_{\mathbf{k}}$ ),

$$
\rho_{0}\left(y, y^{\prime}\right) \longrightarrow \rho_{\xi}\left(y, y^{\prime}\right)=\rho_{0}\left(y, y^{\prime}\right) \exp \left(-\frac{\xi}{2}\left(y-y^{\prime}\right)^{2}\right) .
$$

Here, the parameter $\xi$ encodes the details of the interaction between the modes and their environment. Given a specific model with a specific interaction, $\xi$ can be calculated. The special decoherence process (47) is typical for the description of localization in quantum mechanics $15,17,22$.

One recognizes from (48) that interferences between different values of the field amplitude $y$ have been suppressed by interaction with the environment. This is decoherence. So far we have just assumed without derivation that $|y\rangle$ is the pointer basis, that is, the relevant robust system basis which is distinguished by the environment. This must, of course, be justified. A detailed derivation for the field-amplitude basis to be the pointer basis has been presented in ${ }^{21}$ and ${ }^{25}$. We review here the main arguments and refer the reader to these references for more details.

According to the classical equations, for modes with very large wavelength one has $y \propto a$, that is, the physical fluctuations $\delta \phi$ are approximately constant ('frozen'). In the Heisenberg picture of the quantum theory, this 
means that the operator $\widehat{\delta \phi}$ approximately commutes with the Hamiltonian. Now comes the crucial point. Additional (environmental) fields coupling with the cosmological fluctuations are expected to couple field amplitudes, not canonical momenta of field amplitudes; that is, the coupling is expected to involve $\widehat{\delta \phi}$, not its momentum. Consequently, the fluctuations $\widehat{\delta \phi}$ commute with the whole Hamiltonian of system plus environment. Such a variable is a pointer observable par excellence $\frac{15,16,17}{}$. It is stable (robust) in time because of this commutativity which holds for the wavelengths much bigger than the Hubble scale. The phenomenological expectation (48) is thus fully justified. One must keep in mind, though, that $\widehat{\delta \phi}$ is only an approximate pointer observable: although the non-diagonal terms in (48) become exponentially suppressed, they never vanish exactly, as would be the case if the $\widehat{\delta \phi}$ were the exact pointer observable. In fact, the reduced density matrix can be decomposed into narrow Gaussians in $\delta \phi$-space. The whole situation is in strong analogy to the localization of a massive particle by scattering with the environment $15,17,22$.

The approximate commutativity of $\widehat{\delta \phi}$ with the full Hamiltonian means in particular that the kinetic term, that is, the $p^{2}$-term, of the system becomes irrelevant in the large-squeezing limit. If this term were relevant (as it is for modes with smaller wavelength), the pointer basis would not be the field-amplitude basis, but the coherentstate basis $\stackrel{25}{2}$. But this is not the case here. The coherentstate basis is, in particular, unstable under the time evolution.

So far we have restricted our attention to a special initial state: the vacuum state. This is, however, not necessary. $\operatorname{In}^{25}$ we have presented a formalism that is general enough to encompass a wide range of initial states and interactions. A central role in this formalism is played by a master equation for the reduced density matrix, which is of the Lindblad form. More concretely, the density matrix is assumed to satisfy 15

$$
\frac{\mathrm{d} \hat{\rho}}{\mathrm{d} t}=-\mathrm{i}[\hat{H}, \hat{\rho}]+\hat{L} \hat{\rho} \hat{L}^{\dagger}-\frac{1}{2} \hat{L}^{\dagger} \hat{L} \hat{\rho}-\frac{1}{2} \hat{\rho} \hat{L}^{\dagger} \hat{L},
$$

where $\hat{L}$ is the Lindblad operator. Most of the particular models discussed in the literature lead to a master equation of this form. It is thus of interest to study this equation as general as possible. We have assumed that the Lindblad operator is linear in our variables $p$ and $y$, but kept it general otherwise. The Hamiltonian $\hat{H}$ is given by the expression (17).

The results of our discussion in 25 can be summarized as follows. It turns out that the behaviour of the master equation is qualitatively different for modes outside the Hubble radius (as is the case here) and the modes inside. The decoherence time $t_{\mathrm{d}}$ for the modes with wavelengths much bigger than the Hubble radius is during inflation of the order

$$
t_{\mathrm{d}} \sim H_{\mathrm{I}}^{-1} \ln \frac{H_{\mathrm{I}}^{-1}}{t_{0}}
$$

where $H_{\mathrm{I}}$ is the (approximately constant) Hubble parameter during inflation, and $t_{0}$ is a typical time characteristic for the details of the interaction. We emphasize that (50) is approximately independent of these details. It is basically given by the Hubble time, with the details only entering logarithmically. The time $t_{\mathrm{d}}$ also gives the timescale for the Wigner function to become positive. The reduced density matrix can then be decomposed into an apparent ensemble of narrow Gaussians for the values

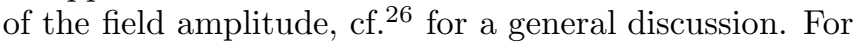
the large-wavelength modes in the radiation-dominated phase one obtains instead

$$
t_{\mathrm{d}} \sim \frac{H_{\mathrm{I}} t_{\mathrm{L}}^{2}}{2}
$$

where $t_{\mathrm{L}}$ depends again on the details of the interaction. One has now a more sensitive dependence on the interaction. Moreover, for $H_{\mathrm{I}} t_{\mathrm{L}} \gg 1$ one has a much longer decoherence time than during inflation. This means that, depending on the interaction, decoherence can be much less efficient than during inflation.

For modes smaller than the Hubble scale, the situation is very different 25 . Taking as a representative example a photon bath as the environment (realized e.g. by the $\mathrm{CMB}$ ), the decoherence time is independent of the Hubble parameter and strongly dependent on the coupling to the bath. Dissipation now becomes the dominant source of influence, in contrast to the case of the super-Hubble modes for which only entanglement occurs.

Decoherence is often connected with symmetry breaking $\frac{15}{2}$, see also $\frac{27}{2}$, section 6.1 . This is also the case here. The initial de Sitter-invariant vacuum state for the fluctuations is highly symmetric. But the observed classical fluctuations are certainly non-symmetric. This can easily be understood and does not require new physics (as e.g. demanded in 28 ). The initial vacuum state develops into a squeezed vacuum, which can be understood as a superposition of different field-amplitude eigenstates. Decoherence then makes this indistinguishable from an ensemble of (approximate) field-amplitude eigenstates, each of which is highly inhomogeneous. The situation resembles the case of spontaneous symmetry breaking in field theory, where the symmetric initial state evolves into a superposition of 'false vacua'. After decoherence one is left with an apparent ensemble of different false vacua, one of which corresponds to our observed world.

\section{B. Entropy}

In Sec. III the primordial fluctuations were treated as isolated and thus described by a pure (squeezed) state. Consequently, they possess zero entropy: all information is contained in the system itself. But as we have seen, the primordial fluctuations are an open quantum system; they are entangled with their environment. Because of this entanglement, the fluctuations are described by the reduced density matrix (48). They thus possess positive 
entropy because the information about the correlations with the environment are unavailable in the system itself. The local entropy is calculated from the standard von Neumann formula,

$$
S=-\operatorname{tr}\left(\rho_{\xi} \ln \rho_{\xi}\right),
$$

where $\rho_{\xi}$ is given in (48), and where we have set $k_{\mathrm{B}}=1$. Considering one (real) mode with wave number $k$, the maximal entropy, $S_{\max }$, would be $2 r_{k}$, where $r_{k}$ is again the squeezing parameter 29 (we skip again the index $k$ in the following). We have calculated and discussed the entropy for the fluctuations in 23,25 . To display the result, it is convenient to introduce the dimensionless parameter $\chi=\xi / \Omega_{\mathrm{R}}$, where $\Omega_{\mathrm{R}}$ is the width of the Gaussian (29); it controls the strength of decoherence. (In the case of pure exponential inflation one has $\chi=\xi\left(1+4 \sinh ^{2} r\right) / k$.) Inserting (48) into (52), one gets the explicit expression ${ }^{25}$

$$
\begin{aligned}
S & =-\ln \frac{2}{\sqrt{1+\chi}+1}-\frac{1}{2}(\sqrt{1+\chi}-1) \ln \frac{\sqrt{1+\chi}-1}{\sqrt{1+\chi}+1} \\
& =\ln \frac{1}{2} \sqrt{\chi}-\sqrt{1+\chi} \ln \frac{\sqrt{1+\chi}-1}{\sqrt{\chi}} .
\end{aligned}
$$

One recognizes that the entropy vanishes for $\xi \rightarrow 0$, as it must for a pure state. In the limit $\chi \gg 1$ (large decoherence) one gets

$$
S=1-\ln 2+\frac{\ln \chi}{2}+\mathcal{O}\left(\chi^{-1 / 2}\right)
$$

This asymptotic value is readily attained.

As we have emphasized above, modes with wavelength bigger than the Hubble scale can only experience pure entanglement, not direct disturbance. In such a case the entropy obeys the bound

$$
S<\frac{S_{\max }}{2}=r .
$$

The same bound follows from the general discussion of the Lindblad equation ${ }^{25}$. It can also be interpreted in the following way ${ }^{25}$ : in spite of decoherence, some squeezing compared to the vacuum state (which has $\Omega_{\mathrm{R}}=k$ ) remains. In the language of the Wigner function it means that the Wigner ellipse is not smeared out to become a circle, but still exhibits an elongated and a squeezed part. And this has important consequences for observation! If the bound (55) were violated, there would no longer be any coherences between the field amplitude and the momentum and, consequently, no coherences in the coupled baryon-photon plasma (Sec. IV). There would then not be any acoustic peaks in the anisotropy spectrum of the CMB - in contrast to observation! The fundamental questions of the quantum-to-classical transition have thus observational relevance.

The upper bound $S_{\max } / 2$ corresponds to the case when the pointer basis is the exact field-amplitude basis. (For $S=S_{\max }$, the pointer basis would be the particle-number basis.) As our pointer basis consists of narrow packets in field amplitudes, the entropy of the fluctuations approaches the upper bound asymptotically.

The existence of the bound (55) shows, again, how peculiar the case of fluctuations in an inflationary universe is. According to a theorem by $\operatorname{Page}^{30}$ (see also ${ }^{31}$ ), if a total quantum system with dimension $m n$ is in a random pure state, the average entropy of a subsystem of dimension $m \leq n$ is almost maximal. But this is not the case for our system: the situation for the fluctuations during inflation is very special, and their entropy cannot exceed half of the maximal entropy, which leaves enough information for the formation of the acoustic peaks.

Our results for the entropy in ${ }^{23}$ and $^{25}$ also yield the following simple formula for the entropy production during inflation:

$$
\dot{S} \approx \dot{r} \approx H_{\mathrm{I}}
$$

For chaotic systems, the entropy production rate is proportional to the Lyapunov parameter. This would correspond in our case to the Hubble parameter $H_{\mathrm{I}}$. However, our system is not chaotic, but only classically unstable, so the analogy is not complete.

Using (50), one can find the amount of entropy produced after the decoherence time $t_{\mathrm{d}}$,

$$
S \sim H_{\mathrm{I}} t_{\mathrm{d}} \sim \ln \frac{H_{\mathrm{I}}^{-1}}{t_{0}} .
$$

In the radiation-dominated phase following inflation, a relation similar to (56) holds, with $H_{\text {I }}$ replaced by the Hubble parameter $H \propto t^{-1}$. The entropy thus only increases logarithmically in time, not linearly as in inflation.

\section{Specific models}

So far, we have kept the discussion as general as possible. We have reviewed the arguments which lead to the result that cosmological fluctuations appear like a classical ensemble of field amplitudes. Necessary requirements are the inflationary expansion of the universe and the focus on modes that are highly squeezed. An interaction with some environment is needed, but the details of it are unimportant. Still, it is of interest to discuss specific examples for such interactions. Our paper ${ }^{25}$ gives an extended list of references; here we shall restrict ourselves to some recent examples.

The purely spatial entanglement between the modes inside the Hubble scale and outside the Hubble scale was discussed $\mathrm{in}^{32}$, see also ${ }^{33}$. It was shown there that this entanglement is, by itself, sufficient to produce the desired decoherence. This is analogous to the black-hole case where the decoherence from the tracing out of the modes behind the horizon leads to the thermal radiation of the Hawking effect ${ }^{18,19}$. The authors of ${ }^{32}$ also showed that the entropy scales with the volume inside 
the Hubble scale and satisfies an upper bound of $S \approx r$ per mode, which coincides with the upper bound (55) discussed above. It is thus not in conflict with the observed acoustic peaks in the cosmic microwave background.

Instead of pure spatial entanglement one can consider the entanglement of our strongly squeezed super-Hubble modes with sub-Hubble modes (which then play the role of the environment). This was discussed, for example, in 34 . The authors take the short-wavelength modes to be in their ground states and find that decoherence is not sufficient during inflation. This happens because vacuum states are usually ineffective to lead to decoherence 15 . Our arguments above and $i n^{25}$ can thus only be applied to this model if at least some modes are not in their ground states. But such modes can be found: one can interpret the fluctuations with wavelengths $\lambda \gtrsim H_{\mathrm{I}}^{-1}$ as an appropriate environment; they assume a role intermediate between ground state and state with large squeezing. Ideas similar to the ones in ${ }^{34}$ have been pursued in 35,36 , and elsewhere, with results that are consistent with our general discussion above. A variant of this system-environment split is presented in ${ }^{37}$ using a two-field model of inflation. There, the system consists of curvature perturbations, and the environment consists of isocurvature modes. Finally, another possible source of sub-Hubble modes being in non-vacuum states is the secondary gravitational wave background ("foreground" in astronomical terminology) emitted by matter after the end of inflation 23 .

\section{CONCLUSION}

Inflation is a robust scenario which gives an elegant solution to some oustanding problems of Big-Bang cosmology, and its predictions are in agreement with present observations, in particular the accurate CMB anisotropy data. It is gratifying that this scenario offers also the possibility to deal with such fundamental and subtle questions as to why quantum perturbations produced in the early Universe give rise to classical inhomogeneities today. We believe that this aspect is no less fascinating than its other successful predictions.

We expect that models of the quantum-to-classical transition for the primordial fluctuations will continue to appear in the literature. But we are convinced that the general mechanism of this transition presented in this essay will hold true for all scenarios based on inflation.

\section{Acknowledgements}

We are happy to thank our collaborator Alexei Starobinsky for his crucial input in obtaining the results presented here and for his comments on our manuscript.

We kindly acknowledge financial support from The Foundational Questions Institute (http://fqxi.org) for the visit of D. P. to the University of Cologne.
[1] A. R. Liddle and D. H. Lyth, Cosmological inflation and large-scale structure, Cambridge University Press, Cambridge (2000).

[2] L. P. Grishchuk and Y. V. Sidorov, Class. Quantum Grav. 6, L161 (1989).

[3] D. Polarski and A. A. Starobinsky, Class. Quantum Grav. 13, 377 (1996).

[4] J. Lesgourgues, D. Polarski, and A. A. Starobinsky, Nucl. Physics B 497, 479 (1997).

[5] C. Kiefer and D. Polarski, Ann. Phys. (Leipzig) 7, 137 (1998).

[6] A. A. Starobinsky, JETP Lett. 30, 682 (1979).

[7] E. Komatsu, J. Dunkley, M. R. Nolta, C. L. Bennett, B. Gold, G. Hinshaw, N. Jarosik, D. Larson, M. Limon, L. Page, D. N. Spergel, M. Halpern, R. S. Hill, A. Kogut, S. S. Meyer, G. S. Tucker, J. L. Weiland, E. Wollack, and E. L. Wright, arXiv:0803.0547 1 (2008).

[8] D. Baumann and H. Peiris, Cosmological Inflation: Theory and Observations, contribution to this issue.

[9] W. H. Kinney, E. W. Kolb, A. Melchiorri, and A. Riotto, arXiv:0805.2966v2 (2008); J. Lesgourgues, A. A. Starobinsky, and W. Walkenburg, JCAP 0801:010 (2008).

[10] J. Lesgourgues, S. Prunet, and A. A. Starobinsky, Astron. Astrophys. 359, 414 (2000).

[11] A. H. Guth and S.-Y. Pi, Phys. Rev. D 32, 1899 (1985).

[12] D. H. Lyth, Phys. Rev. D 31, 1792 (1985).

[13] A. Albrecht, P. Ferreira, M. Joyce, and T. Prokopec,
Phys. Rev. D 50, 4807 (1994).

[14] D. H. Lyth and D. Seery, Phys. Lett. B 662, 309 (2008).

[15] E. Joos, H. D. Zeh, C. Kiefer, D. Giulini, J. Kupsch, and I.-O. Stamatescu, Decoherence and the appearance of a classical world in quantum theory, second edition, Springer, Berlin (2003). See also www.decoherence.de.

[16] W. H. Zurek, Rev. Mod. Phys. 75, 715 (2003).

[17] M. Schlosshauer, Decoherence and the Quantum-toClassical Transition, Springer, Berlin (2008).

[18] W. Israel, Phys. Lett. A 57, 107 (1976).

[19] C. Kiefer, Class. Quantum Grav. 18, L151 (2001).

[20] M. Schlosshauer, arXiv:quant-ph/0605249v3 (2008).

[21] C. Kiefer, D. Polarski, and A. A. Starobinsky, Int. J. Mod. Phys. D 7, 455 (1998).

[22] E. Joos and H. D. Zeh, Z. Phys. B 59, 223 (1985).

[23] C. Kiefer, D. Polarski, and A. A. Starobinsky, Phys. Rev. D 62, 043518 (2000).

[24] C. Kiefer, J. Lesgourgues, D. Polarski, and A. A. Starobinsky, Class. Quantum Grav. 15, L67 (1998).

[25] C. Kiefer, I. Lohmar, D. Polarski, and A. A. Starobinsky, Class. Quantum Grav. 24, 1699 (2007).

[26] L. Diósi and C. Kiefer, J. Phys. A 35, 2675 (2002).

[27] H. D. Zeh, The Physical Basis of the Direction of Time, fifth edition, Springer, Berlin (2007).

[28] A. Perez, H. Sahlmann, and D. Sudarsky, Class. Quantum Grav. 23, 2317 (2006).

[29] T. Prokopec, Class. Quantum Grav. 10, 2295 (1993).

[30] D. N. Page, Phys. Rev. Lett. 71, 1291 (1993). 
[31] P. Hayden, D. W. Leung, and A. Winter, arXiv:quant-ph/0407049 2 (2005).

[32] J. W. Sharman and G. D. Moore, JCAP 0711:020 (2007).

[33] Y. Nambu, Phys. Rev. D 78, 044023 (2008).

[34] C. P. Burgess, R. Holman, and D. Hoover, Phys. Rev. D 77,063534 (2008).
[35] P. Martineau, Class. Quantum Grav. 24, 5817 (2007).

[36] F. C. Lombardo and D. López Nacir, Phys. Rev. D 72, 063506 (2005).

[37] T. Prokopec and G. I. Rigopoulos, JCAP 0711:029 (2007). 